\title{
Gaussian beam evolution in nonlinear inhomogeneous fibers
}

\author{
P. Berczyński, ${ }^{* 1}$ Yu.A. Kravtsov ${ }^{2}$ \\ ${ }^{1}$ Institute of Physics, West Pomeranian University of Technology, Szczecin 70-310, Poland, \\ ${ }^{2}$ Institute of Physics, Maritime University of Szczecin, Szczecin 70-500, Poland.
}

Received March 03, 2012; accepted March 20, 2012; published March 31, 2012

\begin{abstract}
The paper analyzes the Gaussian beam (GB) evolution in nonlinear fibers in the framework of paraxial complex geometrical optics (PCGO). This method reduces the problem of Gaussian beam diffraction in inhomogeneous and nonlinear media to the system of the first order ordinary differential equations for the complex curvature of the wave front and for GB amplitude, which can be readily solved both analytically and numerically. As a result, PCGO radically simplifies the description of Gaussian beam diffraction and self-focusing effects as compared to the other methods of nonlinear optics such as: variational method approach, method of moments and beam propagation method. It is shown that the PCGO method readily supplies the solution of Nonlinear Schrödinger Equation (NLS) for self-focusing fiber with a focusing refractive profile.
\end{abstract}

A surprising feature of paraxial complex geometrical optics (PCGO) is its ability to describe the diffraction of a Gaussian beam (GB) [1]-[6]. Recently, the PCGO method has been generalized for nonlinear media of the Kerr type reproducing the basic results of classical nonlinear optics [7] and opening the way for further generalizations.

The present paper studies the complicated behaviour of a GB in nonlinear and transversely inhomogeneous fibers in a very simple mathematical way. The paper presents GB evolution in nonlinear fibers of the Kerr type, where refractive effects and the phenomenon of initial curvature of the wave front are taken into account. Obtained solutions generalize the results of previous papers [8]-[14] in the scope of light beam diffraction and self-focusing in nonlinear fibers.

Let us consider the medium of cylindrical symmetry, which electric permittivity can be presented as a sum of linear and nonlinear part depending on the beam intensity $|u|^{2}$

$$
\varepsilon(\mathbf{r})=\varepsilon_{L}(\mathbf{r})+\varepsilon_{N L}(\mathbf{r})=\varepsilon_{0}+g(\mathbf{r}) \pm\left|\varepsilon_{N L} \| u(\mathbf{r})\right|^{2} .
$$

The permittivity profile in Eq. (1) models nonlinear optical fiber, where the parameter $\varepsilon_{0}=\varepsilon(\eta=0)$ is the permittivity measured along the fiber axis, $\mathbf{r}$ is the radius vector in cylindrical coordinates, the function $g(\mathbf{r})= \pm \eta^{2} / L^{2}$ models the refractive profile of the fiber, where the positive sign describes the defocusing medium and

\footnotetext{
*E-mail: pawel.berczynski@ps.pl
}

the negative sign corresponds to the focusing fiber. The parameter $\eta=\sqrt{x^{2}+y^{2}}$ is the distance from the axis $\mathrm{z}$ and the parameter $\mathrm{L}$ denotes characteristic inhomogeneity scale. The positive value of the nonlinear part of the electric permittivity $\varepsilon_{N L}(\mathbf{r})=\left|\varepsilon_{N L}\right||u(\mathbf{r})|^{2}$ corresponds to a self-focusing fiber and the negative sign of $\varepsilon_{N L}(\mathbf{r})=-\left|\varepsilon_{N L} \| u(\mathbf{r})\right|^{2}$ describes the self-defocusing process.

For an axially symmetric wave beam in an axially symmetric nonlinear medium the PCGO method suggests a solution of the form

$$
u(\eta, z)=A \exp \left(i k_{0} \psi\right)=A(z) \exp \left[i k_{0}\left(\sqrt{\varepsilon_{0}} z+B(z) \eta^{2} / 2\right)\right] .
$$

The real and imaginary parts of the complex curvature $B=B_{R}+i B_{I}$ determine the real curvature $\kappa$ of the wave front and the beam width $w$ correspondingly:

$$
B_{R}=\sqrt{\varepsilon_{0}} \kappa, \quad B_{I}=\frac{1}{k_{0} w^{2}},
$$

where $k_{0}=2 \pi / \lambda_{0}$ and $\lambda_{0}$ is the wavelength of the beam in vacuum. The eikonal equation:

$$
(\nabla \psi)^{2}=\varepsilon
$$

in $(\eta, \mathrm{z})$ coordinates takes the form:

$$
\left(\frac{\partial \psi}{\partial \eta}\right)^{2}+\left(\frac{\partial \psi}{\partial z}\right)^{2}=\varepsilon(z, \eta)
$$

In accordance with paraxial approximation radius $\eta$ should be small enough. Therefore permittivity $\varepsilon(z, \eta)$ in Eq. (5) can be expanded in a Taylor series in $\eta$ in the vicinity of symmetry axis $\mathrm{z}$ :

$$
\varepsilon(z, \eta)=\varepsilon(\eta=0)+\left(\left.\frac{\partial \varepsilon}{\partial \eta}\right|_{\eta=0}\right) \eta+\left(\left.\frac{\partial^{2} \varepsilon}{\partial \eta^{2}}\right|_{\eta=0}\right) \frac{\eta^{2}}{2} .
$$

Substituting eikonal from Eq. (2) and permittivity expansion (6) into eikonal equation (5) and comparing coefficients of $\eta^{0}, \eta$ and $\eta^{2}$, we obtain the relations 


$$
\varepsilon(\eta=0)=\varepsilon_{0},\left.\quad \frac{\partial \varepsilon}{\partial \eta}\right|_{\eta=0}=0
$$

and the Riccati equation for complex curvature B [6]:

$$
\sqrt{\varepsilon_{0}} \frac{d B}{d z}+B^{2}=\alpha
$$

Parameter $\alpha$ for an axially symmetric medium equals:

$$
\alpha=\left.\frac{1}{2} \frac{\partial^{2} \varepsilon}{\partial \eta^{2}}\right|_{\eta=0}
$$

Substituting (3) in Eq. (2), we obtain a Gaussian beam of the form

$$
u(\eta, z)=A(z) \exp \left(-\frac{\eta^{2}}{2 w^{2}}\right) \exp \left[i k_{0}\left(\sqrt{\varepsilon_{0}} z+\kappa \frac{\eta^{2}}{2}\right)\right] .
$$

Solution (10) reflects the general feature of PCGO, which in fact deals with Gaussian beams. The general form of the Gaussian beams in the 3D inhomogeneous media, as well as a general Riccati equation for complex curvature parameter $B$ can be found in [6] and in the review paper [3].

In the framework of paraxial approximation the amplitude $\mathrm{A}=\mathrm{A}(\mathrm{z})$ is complex-valued and satisfies the transport equation

$$
\operatorname{div}\left(A^{2} \nabla \psi\right)=0,
$$

which for axially symmetric beam in $(\eta, \mathrm{z})$ coordinates takes the following form.

$$
\frac{d A^{2}}{d z} \frac{\partial \psi}{\partial z}+\left[\frac{1}{\eta} \frac{\partial}{\partial \eta}\left(\eta \frac{\partial \psi}{\partial \eta}\right)+\frac{\partial^{2} \psi}{\partial z^{2}}\right] A^{2}=0
$$

In paraxial approximation assuming that $\eta$ is small parameter equation (12) reduces to the ordinary differential equation in the form:

$$
\sqrt{\varepsilon_{0}} \frac{d A}{d z}+B A=0 .
$$

The above equation for GB complex amplitude, as well as the Riccati equation for complex curvature $\mathrm{B}$ are the basic PCGO equations. PCGO reduces the problem of GB diffraction to the domain of ordinary differential equation. Having calculated the complex parameter B from Riccati equation (8), one can readily determine complex amplitude A by integration of equation (13). As a result, the complex amplitude of cylindrically symmetric GB takes the form

$$
A\left(z^{\prime}\right)=A_{0} \exp \left(-\int B\left(z^{\prime}\right) d z^{\prime}\right)
$$

where $A_{0}=A(0)$ is an initial amplitude and $z^{\prime}=z / \sqrt{\varepsilon_{0}}$.
Riccati equation (8) is equivalent to the set of two equations for the real and imaginary parts of the complex curvature $B$. This set of equations together with (3) leads to the relation between the beam width $w$ and the wave front curvature $\kappa$

$$
\kappa=\varepsilon_{0} \frac{1}{w} \frac{d w}{d z}
$$

and ordinary differential equation of the second order for GB width evolution, which for nonlinear inhomogeneous fibers of the Kerr type takes the following form

$$
\varepsilon_{0} \frac{d^{2} w}{d z^{2}}-\left(\alpha_{N L}+\alpha_{g}\right) w=\frac{1}{k_{0}^{2} w^{3}},
$$

where

$$
\begin{gathered}
\alpha_{N L}=\left.\frac{1}{2} \frac{d^{2}}{d \eta^{2}}\left( \pm\left|\varepsilon_{N L}\right||u|^{2}\right)\right|_{\eta=0}=\mp \frac{\left|\varepsilon_{N L}\right| A_{0}^{2} w_{0}^{2}}{w^{4}(z)}, \\
\alpha_{g}=\left.\frac{1}{2} \frac{d^{2} g}{d \eta^{2}}\right|_{\eta=0}= \pm \frac{1}{L^{2}} .
\end{gathered}
$$

For nonlinear self-focusing fiber with a defocusing permittivity profile, Eq. (16) provides the following solution for $\mathrm{GB}$ width evolution:

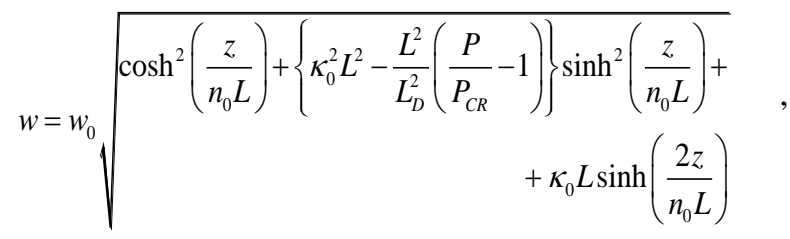

where $P=\frac{1}{8} c n_{0} w_{0}^{2}\left|A_{0}\right|^{2}$ is the total beam power, $P_{C R}=\frac{1}{8} \frac{c n_{0}}{k_{0}^{2}\left|\varepsilon_{N L}\right|}$ is the critical power, $L_{D}=k_{0} w_{0}^{2}$ is the Rayleigh diffraction length and refractive index $n_{0}=\sqrt{\varepsilon_{0}}$.

From Eq. (19) one can notice that when the total beam power is smaller than critical power $P<P_{C R}$ for both positive $\kappa_{0}>0$ and negative $\kappa_{0}<0$ curvature of the wave front, the GB width increases. When the total beam power is greater than the critical power and smaller than some characteristic power $P_{C R}<P<P_{C R}\left[1+\frac{L_{D}^{2}}{L^{2}}\left(\kappa_{0} L+1\right)^{2}\right]$ GB width still increases taking into account also the sign and the value of the initial wave front curvature. When the total beam power is equal to the characteristic power 
$P=P_{C R}\left[1+\frac{L_{D}^{2}}{L^{2}}\left(\kappa_{0} L+1\right)^{2}\right]$ the solution (19) reduces to the form

$$
w=w_{0} \sqrt{1+\kappa_{0} L\left[1-\exp \left(-\frac{2 z}{n_{0} L}\right)\right]} \text {. }
$$

At last, when the total beam power is greater than characteristic power $P>P_{C R}\left[1+\frac{L_{D}^{2}}{L^{2}}\left(\kappa_{0} L+1\right)^{2}\right]$ the GB collapses regardless of the sign and value of the initial wave front curvature. For the case when $\kappa_{0}=0$ the characteristic power determines self-trapping power of the beam:

$$
P^{\prime}=P_{C R}\left(1+L_{D}^{2} / L^{2}\right)
$$

for which one obtains a stationary solution. One can notice that self-trapping power (21) is greater than the critical power $P^{\prime}>P_{C R}$ due to the presence of defocusing refraction.

For self-defocusing fiber with a focusing permittivity profile Eq. (16) supplies the solution

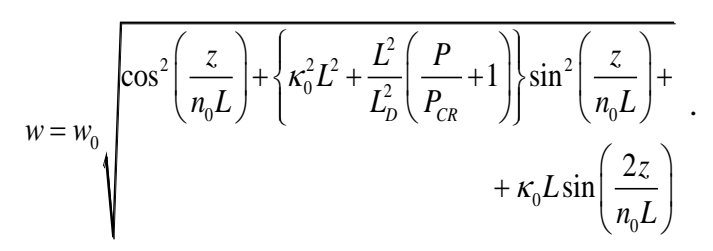

From Eq. (22) one can notice that the GB is bounded for both sub-critical $P<P_{C R}$ and over-critical power $P \geq P_{C R}$. For the case when $L<L_{D}$ and $\kappa_{0}=0$, one obtains a stationary solution when the total beam power is equal to self-trapping power, which for the case of selfdefocusing fiber with focusing permittivity has the form

$$
P^{\prime \prime}=P_{C R}\left(L_{D}^{2} / L^{2}-1\right) \text {. }
$$

For self-focusing fiber with a focusing permittivity profile Eq. (16) provides the result

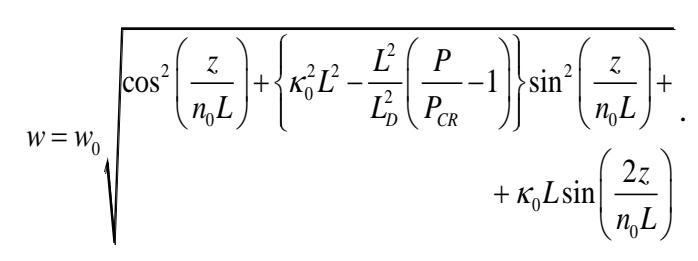

An identical analytical solution has been obtained for GB with the initially plane wave front $\kappa_{0}=0$ in the framework of NLS by the variational method approach [11]. From solution (24), one can notice that for the subcritical power $P<P_{C R}$ the GB is bounded for both: $\kappa_{0}>0$ and $\kappa_{0}<0$. For the GB power which is greater than the critical power $P>P_{C R}$ and smaller than the characteristic power:

$$
P<P_{C R}\left[1+\kappa_{0}^{2} L_{D}^{2}\right]
$$

the positive value of the initial curvature of the wave front $\kappa_{0}>0$ eliminates the collapse effect. When the total beam power $P$ is greater than the characteristic power $P>P_{C R}\left[1+\kappa_{0}^{2} L_{D}^{2}\right]$ the GB always collapses. In the case when $\kappa_{0}=0$, the presence of focusing refraction determines the self-trapping power, which is smaller than the critical power and is equal to:

$$
P^{\prime \prime \prime}=P_{C R}\left(1-L_{D}^{2} / L^{2}\right) \text {. }
$$

\section{References}

[1] Yu. A. Kravtsov, G.W. Forbes, A.A. Asatryan, in Progress in Optics, Ed. E.Wolf, 39, 3 (Amsterdam, Elsevier 1999).

[2] S.J. Chapman, J.M. Lawry, J.R. Ockendon, and R.H. Tew, SIAM Review 41, 417 (1999).

[3] Yu.A. Kravtsov, P. Berczynski. Stud. Geophys. Geod. 51(1), 1 (2007).

[4] Yu.A. Kravtsov, Geometrical Optics in Engineering Physics (Alpha Science International, UK 2005)

[5] P. Berczynski, Yu.A. Kravtsov. Physics Letters A 331(3-4), 265 (2004).

[6] P. Berczynski, K. Yu. Bliokh, Yu. A. Kravtsov, A. Stateczny, J. Opt. Soc. Am. A 23(6), 1442 (2006).

[7] P. Berczynski, Yu.A. Kravtsov, A.P. Sukhorukov. Physica D: Nonlinear Phenomena 239/5, 241 (2010).

[8] S.A. Akhmanov, R.V. Khokhlov, A.P. Sukhorukov, Laser Handbook, vol. 2 (Elsevier 1972).

[9] J.A. Arnaud, Beams and Fiber Optics (New York, Academic Press 1976).

[10] G. Agrawal, Nonlinear Fiber Optics (New York, Academic Press, 1989).

[11] J.T. Manash, P.L. Baldeck, R.R. Alfano, Opt. Lett. 13(7), 589 (1988).

[12] M. Karlsson, D. Anderson, M. Desaix, Opt. Lett. 17(1), 22 (1992).

[13] B.A. Malomed, Prog. in Opt. 43, 69 (2002).

[14] S. Longhi, D. Janner, J. Opt. B. 6 S303 (2004). 